\title{
Biomodulatory potential of low-level laser on neoangiogenesis and remodeling tissue. A literature review
}

\section{Tila Fortuna', Ana Cristina Gonzalez ${ }^{2}$, Maíra Sá Ferreira' ${ }^{3}$, Sílvia Reis ${ }^{4}$, Alena Medrado ${ }^{5}$}

'Corresponding author. Master's in Odontology. Salvador, Bahia, Brazil. tilafortuna@gmail.com ${ }^{2} \mathrm{PhD}$ in Imunology. Researcher at the Gonçalo Moniz Research Centre - FIOCRUZ. Salvador, Bahia, Brazil. acogonzalez@hotmail.com ${ }^{3}$ Undergratuate student at BAHIANA - School of Medicine and Public Health. Salvador, Bahia, Brazil. mairasa@yahoo.com.br ${ }^{4} \mathrm{PhD}$ in Odontology. Professor at BAHIANA - School of Medicine and Public Health. Salvador, Bahia, Brazil. srareis@uol.com.br ${ }^{5} \mathrm{PhD}$ in Human Pathology. Professor at BAHIANA - School of Medicine and Public Health. Salvador, Bahia, Brazil. alenamedrado@hotmail.com

Abstract I Introduction: In recent years, cellular biostimulation induced by low-level laser therapy has been widely studied. Among some of the processes stimulated by low-level laser are cell cycle, phenotypic modulation as well as vascular changes, such as vasodilation and neoangiogenesis. The increased number of new blood vessels accelerates inflammation, and also increases wound healing. Neoangiogenesis is still an important process of tissue repair, since it always precedes collagen synthesis and matrix extracellular remodeling. Increasing scientific evidence has shown that photobiomodulation stimulates neoangiogenesis, fibroblast proliferation and collagen biosynthesis. Objective: Review the literature about the effects of low level laser therapy on neoangiogenesis and fibroplasia and describe how these processes occur in the tissue subjected to an injury. Methods and Matereials: The search tool used was the Medline / Pubmed and the following keywords were used: remodeling, wound healing, neoangiogenesis, fibroplasia, laser therapy, low-level laser. Manuscripts published from 1990 to 2017 were selected. Results: Increasing scientific evidence has shown that photobiomodulation stimulates neoangiogenesis, fibroblast proliferation and collagen biosynthesis. Conclusion: Neoangiogenesis always precedes collagen synthesis and matrix extracellular remodeling. However, little is known of the action of laser therapy in the late stages of tissue repair.

Keywords: Low-Level Light Therapy; wound healing; extracellular matrix; collagen. 


\section{Introduction}

Tissue repair process is a physiological response to injury, which usually results in total or partial restoration of tissue morphology and function. The healing process begins with coagulation and acute inflammation, which is essentially characterized by vasodilation and increased vascular permeability. These events are triggered by potent preformed chemical mediators in secretory granules such as histamine, which is a vasoactive amine released by mast cells ${ }^{1}$. Early in the acute phase of inflammation, a new microvasculature is reconstructed to restore the supply of nutrients for tissue formation, preventing hypoxia and characterizing the beginning of the proliferative phase ${ }^{2}$. The increased number of new blood vessels accelerates the development of inflammation, neoangiogenesis and promotes tissue remodeling. The non-occurrence of this important biological phenomenon will result in delayed wound healing and/or deficient biosynthesis of the elements that integrate the new extracellular matrix ${ }^{2,3}$.

Vascular dynamics is a highly orchestrated process. Endothelial cells produce proteolytic enzymes responsible for basement membrane and extracellular matrix degradation. Consequently, cellular migration and formation of new vascular sprouts occurs. Neoangiogenesis establishes itself through the concomitant action of growth and inhibitory factors. Several complex signal transduction pathways are involved in the regulation of this process ${ }^{4}$.

In the later stages of tissue repair, formation of granulation tissue occurs and it becomes progressively less vascularized. This tissue, which may have initiated on the 3rd day following the establishment of the lesion, is a provisional one upon which the future extracellular matrix will be formed.
Gradual deposition of connective fibers and fibrous cicatricial tissue takes place and the reconstructed tissue gradually displays a higher degree of fiber compression, until it finally matures over time ${ }^{5}$.

The effects of angiogenesis and pro-angiogenic factors including the activity of the Vascular Endothelial Growth Factor (VEGF) in fibroplasia, extracellular matrix remodeling and the final phase of cicatricial tissue formation have been studied extensively. It seems that angiogenesis could extend to even longer periods in the tissue, even in the absence of clinical evidence of the initial injuryb. Indeed, in an experimental study that evaluated the regression of granulomatous lesion performed by Andrade et al. ${ }^{7}$, it was demonstrated that vascular tissue remodeling is able to sustain itself for a relatively long period after the establishment of the lesion.

Low-level laser therapy (LLLT), phototherapy or photobiomodulation refers to the use of photons at a non-thermal irradiance to alter biological activity. LLLT uses either coherent light sources (lasers) or non-coherent light sources consisting of filtered lamps or light-emitting diodes (LED) or, on occasion, a combination of both in the range of $1 \mathrm{~mW}$ $500 \mathrm{~mW})^{8,9}$. It is used to promote tissue regeneration, reduce inflammation and relieve pain and it is also defined by several other parameters. The light is typically of narrow spectral width in the red or near infrared (NIR) spectrum (600nm - 1000nm), with a power density (irradiance) between $1 \mathrm{mw}$ $5 \mathrm{~W} / \mathrm{cm}^{2}$ and a dose (power irradiation time/area irradiated) of $10-2$ to $102 \mathrm{~J} / \mathrm{cm}^{2}$. It is typically applied to the injury for a minute or so, a few times a week for several weeks (Table 1). Other significant parameters include a wavelength between 300 and $10,600 \mathrm{~nm}$, a pulse rate of 0 (continuous) to 5,000 $\mathrm{Hz}$, a pulse duration of 1 to 500 milliseconds, an interpulse interval of 1 to 500 milliseconds ${ }^{10,11}$. 
Table 1. Irradiation parameters involved in determining the LLLT dose.

\begin{tabular}{|c|c|c|}
\hline Irradiation parameter & Unit of measurement & Comment \\
\hline Energy & J (Joules) & $\begin{array}{l}\text { Calculated as: Energy }(J)=\text { Power }(W) \times \\
\text { time (s). Using Joules as an expression of } \\
\text { dose is potentially unreliable as it assumes } \\
\text { a reciprocity (the inverse relationship } \\
\text { between power and time). This parameters } \\
\text { ignores Irradiance. }\end{array}$ \\
\hline Energy Density & $\mathrm{J} / \mathrm{cm}^{2}$ & $\begin{array}{l}\text { Common expression of LLLT dose. It mixes } \\
\text { several irradiation parameters and is } \\
\text { potentially unreliable as it assumes a } \\
\text { reciprocity relationship between irradiance } \\
\text { and time. }\end{array}$ \\
\hline Irradiation Time & s & Is one of the irradiation parameters. \\
\hline Treatment interval & Hours, days or weeks & Another important irradiation parameter. \\
\hline
\end{tabular}

Adapted from Huang et al., 2009

Unlike other medical laser procedures, LLLT is not an ablative or thermal mechanism, but rather a photochemical effect comparable to photosynthesis in plants whereby the light is absorbed and exerts a chemical change. The same light source may have opposite effects on the same tissue depending on the parameters used and these conflicting views are probably due to the biphasic effects of light ${ }^{10,11}$.

LLLT acts by inducing a photochemical reaction in the cell, a process referred to as biostimulation or photobiomodulation. Photobiology works on the principle that, when light hits certain molecules called chromophores, the photon energy causes electrons to be excited and jump from low-energy orbits to higher-energy orbits. In nature, this stored energy can be used by the system to perform various cellular tasks, such as photosynthesis and photomorphogenesis ${ }^{12}$.

Although there is a large collection of studies concerning the biological effects induced by lowlevel laser therapy in the early stages of tissue repair $^{13}$, its effects on the extracellular matrix in the late stages of healing have not been widely explored in literature. The beneficial effects of low-level laser have a decisive impact on the primary changes observed in the injured tissue sample vasodilation, stimulating migration of inflammatory cells and increasing lymphatic drainage ${ }^{14,15}$. In respect of the proliferative processes, some authors have reported a significant increase in neoangiogenesis, fibroblast proliferation and collagen biosynthesis ${ }^{16,17}$. However, till now, it is not known for how long the primary beneficial actions described, taking into consideration the cicatricial period of a specific tissue, would extend. Although the answer to this question can vary according to the specific tissue, this aspect should be further evaluated by conducting additional clinical trials and experimental studies.

In the face of the evidence reported in literature, which highlights the importance of neoangiogenesis for tissue remodeling and the participation of the laser in this process, the aim of this current paper is to review the effects of low-level laser therapy on neoangiogenesis and fibroplasia as well as describe how these processes are carried out on damaged tissue.

\section{Liłerature review}

\section{Laser therapy and tissue repair}

Tissue repair consists of four well-defined phases, which, in most cases, occur simultaneously: homeostasis, exudative inflammatory and proliferative phases, and tissue remodeling ${ }^{18}$. Conventionally, the photobiological effects of laser radiation on tissue repair are classified into short and long-term effects. The short-term responses are those in which the effect can be observed a few 
seconds or minutes after irradiation. Observed effects over the long-term are those that occur hours or even days after laser use and usually involve new cell biosynthesis. These effects are especially visible in the proliferative phase of repair ${ }^{17}$.

There are reports in the literature indicating that low-level laser therapy, when applied to skin wounds, is capable of ameloriate inflammation, neoangiogenesis, and epithelial proliferation of fibroblasts, collagen synthesis and deposition, revascularization and wound contraction. In fact, the skin is an excellent tissue to study the effect of laser therapy healing on wound healing 3,14-16,19,20.

Low-level laser therapy accelerates repair because it provides edema reduction and a quick transition from polymorphonuclear inflammatory cell to the monomorphonuclear infiltrate, an increase in the synthesis of collagen fibers and an increase in the organizational pattern of fibrillar components of the extracellular matrix ${ }^{14}$. Lima and colleagues ${ }^{19}$ used 36 Wistar male rats randomly divided in two groups, euthanised at 1,6 and $12 \mathrm{~h}$ after cutaneous surgery in their study. They demonstrated that a 670 $\mathrm{nm}$ gallium aluminum and arsenide (GaAIAs) lowlevel laser modulated inflammation by inhibiting proinflammatory cytokines IL- $1 \beta$ and IL- 6 expression, in periods of 6 to 12 hours after skin injury.

Also, Pereira et al. ${ }^{3}$ investigated the influence of LLLT (GaAlAs $670 \mathrm{~nm}$ ) on mast cells and vascular response to standardized skin wounds in Wistar rats. Their findings corroborate those of Reis et al. ${ }^{14}$. They used thirty-two rats submitted to a wound inflicted with a 6-mm-diameter punch. The animals were divided into four groups: sham treated, those treated with the GaAlAs laser $(670 \mathrm{~nm})$, those treated with dexamethasone $(2 \mathrm{mg} / \mathrm{kg})$, and those treated with both, LLLT and dexamethasone. After 3 and 5 days, the cutaneous wounds were assessed by histopathology. The authors showed significant reduction of edema three days after performing the skin wound in the group treated with laser (compared to the control group). A greater number of degranulated and intact mast cells in the laser group was observed within the first 3 days of the healing process. The authors also found differences in the inflammatory response of the group subjected to laser irradiation. High numbers of polymorphonuclear cells in the early hours of the experiment, followed by the decreasing of this cell infiltrate in the third postoperative day were described.

In 2010, Medrado and colleagues ${ }^{16}$ investigated the effects of laser therapy $(\lambda 670 \mathrm{~nm})$ on pluripotent cells (pericytes) and their role in cutaneous tissue repair in an experimental rat model, over periods of $3,7,10$ and 14 days post-surgery. The authors demonstrated the influence of laser use on cell proliferation and phenotypic modulation of pericytes into myofibroblasts until the 14 th day after performing skin injury.

Rambo et al. ${ }^{20}$ evaluated and compared the effect of LLLT in cutaneous wound healing in young and aged rats. $A$ total of 60 male rats comprising \pm 30 days (young) and 30 aged \pm 500 days (aged) was used. The animals were divided into experimental groups and underwent skin wound and/or treatment with LLLT $(660 \mathrm{~nm})$. Analyses were conducted to verify the effects of LLLT in the tissue repair process after 3,7 and 14 days following experimental skin wound surgical procedure. Gene expression, and protein expression of TNF- $\alpha$, IL- $1 \beta$, and IL-10, obtained in skin wound model were also analyzed. Results showed that there were significant differences between the young control group and the aged control group and their respective treated groups (LLLT young and LLLT aged). Their histological analysis suggested that there were early inflammatory cells, infiltration in laser and control groups of young rats and delayed skin wound healing in laser and control groups of aged rats. Laser also increased the expression of an anti-inflammatory cytokine, IL10 at days 3,7 and 14 of wound healing in both treated groups. However, contrary to these authors, Lima et al. ${ }^{19}$ found a reduction of pro-inflammatory cytokines (IL-1 $\beta$ and IL-6) and anti-inflammatory cytokine IL-10 in a group of young rats treated with low-level laser between 6 and 12 hours after the skin wound had been applied. The authors discussed that although it would be expected a significant increase in IL-10 expression, it not happened under LLLT. They hypothesized that molecular pathways that evoke IL-10 production by macrophages are poorly understood and have not been completely elucidated. Furthermore, they suggested that the release of pro-inflammatory cytokines at the onset of wound healing may prevent the release of other inhibitory chemical mediators such as IL-10. 


\section{Variables of LLLT and its action in the repair}

Correct laser wavelength, dosimetry, irradiance and fluence for each clinical condition are parameters of great significance. Depending on the fluence applied to the tissue surface, for example, different tissue responses can be observed during wound healing ${ }^{21}$. Wavelengths between $632.8 \mathrm{~nm}$ and $1000 \mathrm{~nm}$, have been described as those which present more satisfactory results. Demidova-Rice et al. $^{22}$ in an experimental study on mice, compared different wavelengths $(635,670,720$ and $820 \mathrm{~nm})$ and fluences $\left(1,2,10\right.$, and $\left.50 \mathrm{~J} / \mathrm{cm}^{2}\right)$. A full thickness dorsal excisional wound in mice was performed and treated with a single exposure to light of various wavelengths and fluences 30 minutes after wounding. They found that groups irradiated with $820 \mathrm{~nm}$ wavelength achieved an improvement in wound healing. The authors observed that animals irradiated with $50 \mathrm{~J} / \mathrm{cm}^{2}$ showed a greater expansion of the wound during the first few days and only after 7 to 8 days it returned to its original size. The authors reported that doses of 2 and $10 \mathrm{~J} /$ $\mathrm{cm}^{2}$ showed the greatest benefit of laser therapy in healing, and of these, the dose of $2 \mathrm{~J} / \mathrm{cm}^{2}$ exhibited superior results.

\section{Laser action on angiogenesis}

Angiogenesis is a complex biological process that depends on the balance of pro and anti-angiogenic factors that influence the proliferative state of the endothelium in a wide variety of diseases. In recent years we have witnessed a positive influence of LLLT in the proliferation of endothelial cells during neoangiogenesis ${ }^{15,23}$.

In 1990, Maier et al. ${ }^{24}$ observed the emergence and growth of new larger diameter blood vessels in animals irradiated with low-level laser. Comparing the two groups, the authors found statistically significant differences in irradiated animals compared to the control group, because in the LLLT treated group there was a higher number of newly formed vessels.

Colombo et al. $^{15}$, aimed to evaluate arsenide gallium and aluminum laser $(\lambda 660 \mathrm{~nm})$ effects on the maturation of granulation tissue, focusing on neoangiogenesis, in TGF- $\beta$ expression and biosynthesis of collagen matrix in rodent tissues.
Twenty-four young adult male Wistar rats were randomly distributed in two groups: control and treated with laser. Each group was subdivided into three subgroups according to the animal death timing ( 2,4 and 6 days). The authors concluded that LLLT positively influenced the expression of TGF- $\beta$, corroborating the findings of other authors who also found evidence of increased angiogenesis as a result of laser therapy.

In 2015, Halon and colleagues ${ }^{23}$ aimed to evaluate the rate of neoangiogenesis in wound healing after tooth extraction in HIV positive patients submitted or not submitted to photobiomodulation with a diode laser $(820 \mathrm{~nm})$. Twenty-seven patients with confirmed HIV infection were enrolled in the study ( 6 women, 21 men). Eighty-nine teeth were extracted, 45 sockets were exposed to laser radiation, for five consecutive days following tooth extraction, and the remaining extraction wounds were left to heal spontaneously without laser irradiation. Antigen CD34 was assessed by immunohistochemistry as a marker of angiogenesis, and its expression was examined by computer-assisted histomorphometric image analysis. The authors found a statistically significant difference between laser and control groups in humans infected with HIV. The number of new vessels found in the group of irradiated individuals after six days of surgery, was considerably higher than in the control group. The authors concluded that $\mathrm{CD} 4^{+}$cell number did not influence the process of neoangiogenesis or the effect of laser radiation.

VEGF has been described as an inducer of angiogenesis. The molecular mechanism underlying the stimulation remains unknown, although VEGF can directly stimulate both cells - endothelial cells and fibroblasts ${ }^{6,25}$. In an experiment involving cell signaling, Feng et al. ${ }^{25}$ demonstrated that the proangiogenic factor VEGF was a potent stimulator of vascularization and that LLLT induced the production of VEGF and subsequently neovascularization. However, some authors have suggested that the effect of LLLT on angiogenesis may depend on the laser light wavelength ${ }^{26}$.

In 2006, Tuby et al. ${ }^{27}$ demonstrated stimulation of angiogenesis by VEGF and upregulation of nitric oxide synthesis after using laser light with a wavelength of $\lambda 804 \mathrm{~nm}$. In another experimental study in rats, Pereira et al. $^{3}$ corroborates these 
findings. Surgical procedures were performed on 60 rats divided into sham and laser $(670 \mathrm{~nm})$ groups histological specimens of the laser-treated group presented more extensive areas stained positively with the anti-VEGF antibody 6 to 24 hours after LLLT, compared with the non-irradiated group. However, the authors also reported a reduction in the number of vessels after the first 24 hours of the application of the laser on the skin wound ${ }^{3}$.

\section{Neoangiogenesis and remodeling under laser action}

It is known that LLLT influences the inflammatory response in the first few days following injury, with an increase in the number of polymorphonuclear neutrophils and mast cells, vasodilation, and increased number of blood vessels, thus accelerating the inflammatory process and resulting in faster wound healing ${ }^{3}$. However, little has been studied in relation to low-level laser action in the later stages of healing.
It is known that increased collagen production occurs through photostimulation mechanisms. Certain frequencies and doses can also modulate cellular proliferation and increase the amount of fibroblast growth factors ${ }^{17}$.

In 2008, Medrado et al. ${ }^{17}$ published the results of an experimental study with using the classic model of cutaneous wound healing in rats, which evaluated the photobiomodulated repair process for a period of up to 60 days after performing a standardized skin wound. They showed the biomodulation action of gallium arsenide and aluminum laser $(\lambda 670 \mathrm{~nm})$ on collagen synthesis during different periods of the repair. LLLT produces a better organization of collagen fibers, an important occurrence that assists in generating adequate tensile strength for the new tissue (Figure 1). As a consequence, these authors suggested that this therapeutic modality could efficiently interact with elements of the extracellular matrix (Figure 2). In another study by Mackey et al. ${ }^{28}$, a close association was demonstrated between increased collagen synthesis and regeneration processes that occur in human skeletal muscle after injury.

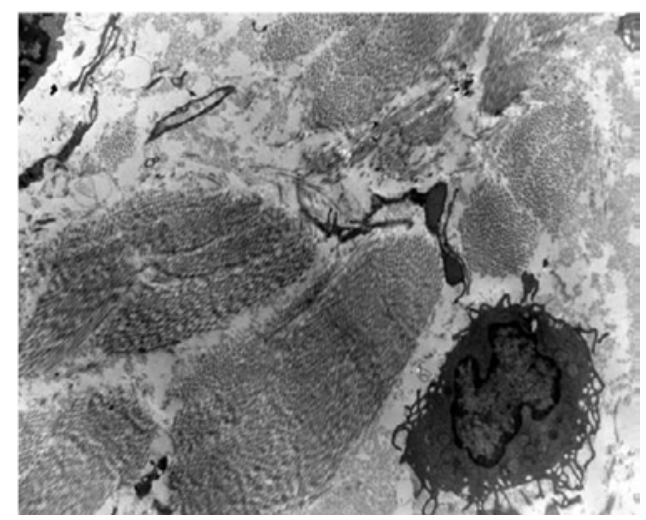

Figure 1. Electron micrograph that illustrates collagen fibers in mouse skin, 10 days after experimental skin injury (4500X magnification).

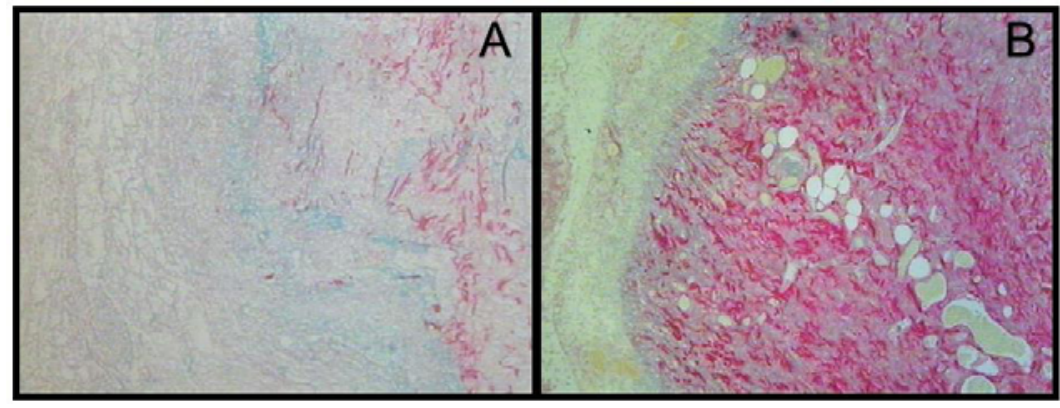

Figure 2. Histological section stained with Sirius red, showing collagen synthesis in rat skin, 72 hours after skin injury without laser irradiation (A) and with low-level laser irradiation (B) (magnification of 100X). 
Baptista et al. ${ }^{5}$ investigated the effects of GaAlAs $(660 \mathrm{~nm})$ laser on collagen type IV in the remodeling process of 45 adult male Wister rats randomly divided into three groups: control $(n=5)$; nontreated cryolesioned group $(n=20)$, and LLLT- ryolesioned group $(n=20)$. Laser irradiation was performed 3 times per week on the injured region. Between 14 and 21 days, the authors observed no significant changes in the type IV collagen distribution pattern between the laser and control groups. However, on the 7th day after the injury, increased immunostaining for collagen type IV was observed in the laser group, when compared to the control and this difference was statistically significant.

\section{Clinical use of LLLT}

Low level laser treatment has increased in popularity and is more frequently used as an adjuvant in the treatment in a various conditions, specially in dentistry ${ }^{29}$.

For instance, the successful placement and integration of the dental implants in the previously grafted extraction sockets require adequate time for the healing and sufficient regeneration of the bone ${ }^{29}$. On their study, Monea et al. ${ }^{29}$, aimed to determine whether LLLT could decrease the time between extraction/socket graft and implant placement, by evaluating histological changes in sockets grafted with a particulate allograft material and treated with LLLT. The authors found that histological results of the site treated with LLLT for 21 days, harvested at 60 days after grafting showed abundant new bone formation without any sign of inflammation. The same results were obtained in the control group not before 120 days post-surgery. So, they assumed that LLLT photobiomodulation can reduce the healing time after grafting the extraction socket. Histological evidence suggests that new bone formation in the sockets appeared within 60 days after LLLT treatment compared to a minimum of 120 days in the control group.

Bisphosphonates are potent inhibitors of osteoclast activity and are used in patients with bone metastases due to malignant diseases or osteoporosis ${ }^{27}$. Walter et al. $^{30}$ evaluated the influence of LLLT effect on bisphosphonate incubated cells, involved in wound healing. They performed an in vitro study to investigate the effect of low level laser $(670 \mathrm{~nm})$ treatment on kera $\neg$ tinocytes, fibroblasts, endothelial cells and osteoblasts treated with clodronate, ibandronate, pamidronate or zoledronate was evaluated. Results showed significant differences of each cell type for each bisphosphonate tested, among the control and experimental groups. It may support the idea of low level laser therapy as a supportive therapy in patients receiving bisphosphonates to prevent and treat bisphospho $\neg$ nate associated osteonecrosis of the jaw.

Merigo et al. $^{31}$ studied fifty-nine patients, who undergo surgical removal of their lower third molars, who were randomly allocated to one of three groups: 17 patients LLLT with traditional drug treatment: 17 patients traditional drug treatment as control group; and 25 patients treated with LLLT only on one side and with traditional drug treatment. LLLT was performed just after the intervention and approximately 12 hours after surgery. They collected all the values of the edema measurements and the Visual Analogue Scale (VAS) reports and performed a statistical analysis. Significant result was obtained for VAS recorded at hospital discharge $(p<0.0001)$. This study demonstrates that LLLT is effective on postoperative pain and edema accelerating healing time and reducing patient's distress.

One of the most common complications of diabetes is the diabetic foot, characterized by the infection, ulceration and/ or destruction of the deep tissues associated to neurological anomalies and in many degrees of the peripheral vascular disease in the lower limb ${ }^{32}$. The ulcer treatment in the diabetic foot requires a multidisciplinary approach, including revascularization and surgical procedures. Feitosa et al. ${ }^{32}$ on their study evaluated the effects of LLLT applying laser on the tissue repair in ulcer carriers due to diabetes. Sixteen type II diabetic patients, ulcer carriers in the lower limbs, participated in the research from which eight were in the control group and eight were submitted to the low-level laser therapy $(632.8 \mathrm{~nm})$. They found a significant decrease in the size of the wound when compared to the control group $(p<0.05)$. The pain was also reported as having an intense improvement in the treated group. Therefore they assumed that low level laser treatment seems to be an efficient method, viable, painless and of low costs concerning the tissue repair ulcers in a diabetic foot. 


\section{Final considerations}

The low-level laser has proved to be capable of promoting the resolution of inflammation, neoangiogenesis, and epithelial proliferation. It also stimulates fibroblast proliferation, collagen synthesis and remodeling, revascularization and wound contraction. Although its effect on the processes that take place in the early stages of tissue repair have been well documented, further studies that consider its influence in the later stages of wound healing are needed.

\section{Competing interests}

No financial, legal or political competing interests with third parties (government, commercial, private foundation, etc.) were disclosed for any aspect of the submitted work (including but not limited to grants, data monitoring board, study design, manuscript preparation, statistical analysis, etc.).

\section{References}

1. Sawasaki I, Geraldo-Martins VR, Ribeiro MS, Marques $M M$. Effect of low-intensity laser therapy on mast cell degranulation in human oral mucosa. Lasers Med Sci. 2009;24(1):1 13-1 16. doi: 10.1007/s10103-007-0531-1

2. Oberringer $M$, Meins $C$, Bubel $M$, Pohlemann $T$. In vitro wounding: effects of hypoxia and transforming growth factor beta 1 on proliferation, migration and myofibroblastic differentiation in an endothelial cell-fibroblast co-culture model. J Mol Histol. 2008;39(1):37-47. doi:10.1007/ s10735-007-9124-3

3. Pereira MC, Pinho CB, Medrado ARP, Andrade ZA, Reis SRA. Influence of $670 \mathrm{~nm}$ low-level laser therapy on mast cells and vascular response of cutaneous injuries. J Photochem Photobio B. 2010;98(3):188-192. doi: 10.1016/i. ¡photobiol.2009.12.005

4. Liu XQ, Mao Y, Wang B, Lu XT, Bai WW, Sun YY et al. Specific matrix metalloproteinases play different roles in intraplaque angiogenesis and plaque instability in rabbits. Plos One. 2014;9(9):1-10. doi: 10.1371/iournal. pone.0107851

5. Baptista AP, Andrade ZA. Angiogenesis and schistosomal granuloma formation. Mem Inst Oswaldo Cruz. 2005;100(2):183-185. doi: 10.1590/S0074$\underline{02762005000200012}$
6. Greaves NS, Ashcroft KJ, Baguneid M, Bayat A. Current understanding of molecular and cellular mechanisms in fibraplasia and angiogenesis during acute wound healing. J Dermatol Scie. 2013;72(3):206-17. doi: 10.1016/i. ¡dermsci.2013.07.008

7. Andrade ZA, Baptista AP, Santana TS. Remodeling of hepatic vascular changes after specific chemotherapy of schistosomal periportal fibrosis. Mem Inst Oswal Cruz 2006;101(supl 1):267-272. doi: 10.1590/S007402762006000900041

8. Chung H, Dai T, Sharma SK, Huang YY, Carroll JD, Hamblin $M R$. The nuts and bolts of low-level laser (light) therapy. Ann Biomed Eng. 201 2;40(2):516-533. doi: 10.1007/s10439011-0454-7

9. Gupta A, Avci P, Sadasivam M, Chandran R, Parizotto $N$, Vecchio $D$ et al. Shining light on nanotechnology to help repair and regeneration. Biotechnol Adv 2012;31(5):607631. doi: 10.1016/i.biotechadv.2012.08.003

10. Huang YY, Chen AC, Carroll JD, Hamblin MR. Biphasic dose response in low level light therapy. Dose Response 2009;7(4):358-383. doi: 10.2203/dose-response.09-027. Hamblin

11. Schindl A, Schindl M, Pernerstorfer-Schon H, Schindl L. Low-intensity laser therapy: a review. J Invest Med 2000;48(5):31 2-26.

12. Hashmi JT, Huang YY, Osmani BZ, Sharma SK, Naeser MA, Hamblin MR. Role of Low-Level Laser Therapy in Neurorehabilitation. Phys Med Rehabil. 2010;2(12 supl 2):S292-S305. doi: 10.1016/i.pmri.2010.10.013

13. Loreti EH, Pascoal VLW, Nogueira BV, Silva IV, Pedrosa DF. Use of laser therapy in the healing process: a literature review. Photomed Laser Surg. 2015;33(2):104-116. doi: $10.1089 /$ pho.2014.3772

14. Reis SRA, Medrado AP, Marchionni AMT, Figueira C, Fracassi LD, Knop LAH. Effect of 670-nm laser therapy and dexamethasone on tissue repair: a histological and ultrastructural study. Photomed Laser Surg. 2008;26(4):307313. doi: $10.1089 /$ pho.2007.2151

15. Colombo F, Valença Neto AAP, de Souza APC, Marchionni AMT, Pinheiro ALB, Reis SRA. Effect of low-level laser therapy $(660 \mathrm{~nm})$ on angiogenesis in wound healing: an immunohistochemical study on a rodent model. Braz Dent J. 2013;24(4):308-312. doi: 10.1590/0103-6440201301867

16. Medrado A, Costa T, Prado T, Reis S, Andrade Z. Phenotype characterization of pericytes during tissue repair following low-level laser therapy. Photoderma Photoimmun Photomedic. 2010;26(4):192-197. doi: $10.1111 /$ i.1600$\underline{0781.2010 .00521 . x}$

17. Medrado AP, Soares AP, Santos ET, Reis SRA, Andrade ZA. Influence of laser photobiomodulation upon connective tissue 
remodeling during wound healing. J Photochem Photobio $B$. 2008;92(3):144-52. doi: 10.1016/i.jphotobiol.2008.05.008

18. Guo S, Dipietro LA. Factors affecting wound healing. J Dent Res. 2010;89(3):219-229. doi: $10.1177 / 0022034509359125$

19. Lima AAM, Spínola LG, Baccan G, Correia K, Oliva $M$, Vasconcelos JF et al. Evaluation of corticosterone and IL-1 $\beta$, IL-6, IL-1 0 and TNF- $\alpha$ expression after 670 $\mathrm{nm}$ laserphotobiomodulation in rats. Lasers Med Sci. 2014;29(2):709-715. doi: 10.1007/s10103-013-1356-8

20. Rambo CSM, Silva Jr JA, Serra AJ, Ligeiro AP, Vieira RP, Albertini $R$ et al. Comparative analysis of low-level laser therapy $(660 \mathrm{~nm})$ on inflammatory biomarker expression during the skin wound-repair process in young and aged rats. Lasers Med Sci. 2014;29(5):1723-1733. doi: $10.1007 /$ s10103-014-1582-8

21. Medrado ARAP, Pugliese LS, Reis SRA, Andrade ZA. Influence of low level laser therapy on wound healing and its biological action upon myofibroblasts. Lasers Surg Med. 2003;32(3):239-244. doi: 10.1002/Ism.10126

22. Demidova-Rice TN, Salomatina EV, Yaroslarsky AN, Herman IM, Hamblin MR. Low-level light stimulates excisional wound healing in mice. Lasers Surg Med. 2007;39(9):706715. doi: $10.1002 / 1 \mathrm{sm} .20549$

23. Halon A, Donizy P, Dziegala $M$, Dobrakowski $R$, Simon $K$. Tissue laser biostimulation promotes post-extraction Neoangiogenesis in HIV-infected patients. Lasers Med Sci. 2015;30:701-706. doi: $10.1007 / \mathrm{s} 10103-013-1411-5$

24. Maier M, Haina D, Landthaler M. Effect of low energy laser on the growth and regeneration of capillaries. Lasers Med Sci. 1990;5(4):381-386. doi: 10.1007/BF02032595

25. Feng J, Zhang $Y$, Xing D. Low-power laser irradiation (LPLI) promotes VEGF expression and vascular endothelial cell proliferation through the activation of ERK/Spl pathway. Cellul Signall. 201 2;24(6): $1116-1125$. doi: 10.1016/i. cellsig.2012.01.013

26. Tachiara R, Farinelli WA, Anderson R. Low intensity lightinduced vasodilation in vivo. Lasers Surg Med. 2002;14:11.

27. Tuby H, Maltz L, Oron U. Modulations of VEGF and INOS in the rat heart by low level laser therapy are associated with cardio protection and enhanced angiogenesis. Lasers Surg Med. 2006;38(7):682-688. doi: 10.1002/Ism.20377

28. Mackey AL, Donnelly AE, Turpeenniemi-Hujanen T, Roper HP. Skeletal muscle collagen content in humans after highforce eccentric contractions. J Appl Physiol. 2004;97(1):197203. doi: 10.1152/iapplphysiol.01174.2003

29. Monea A, Beresescu G, Tibor M, Popsor S, Antonescu DM. Bone healing after low-level laser application in extraction sockets grafted with allograftmaterial and covered with a resorbable collagen dressing: a pilot histological evaluation. BMC Oral Health. 2015;15:134. doi: 10.1186/s12903-015$\underline{0122-7}$

30. Walter C, Pabst AM, Ziebart T. Effects of a low-level diode laser on oral keratinocytes, oral fibroblasts, endothelial cells and osteoblasts incubated with bisphosphonates: An in vitro study. Biomed rep. 2015;3(1):14-18. doi: 10.3892/ $\underline{\text { br.2014.389 }}$

31. Merigo E, Vescovi P, Margali M, Ricotti E, Stea S, Meleti $M$ et al. Efficacy of LLLT in swelling and pain control after the extraction of lower impacted third molars. Laser Ther. 2015;24(1):39-46. doi: 10.5978/islsm.15-OR-05

32. Feitosa MCP, de Carvalho AFM, Feitosa VC, Coelho IM, de Oliveira RA, Arisawa EAL. Effects of the Low-Level Laser Therapy (LLLT) in the process of healing diabetic foot ulcers. Acta Cir Bra. 2015;30(12):852-857. doi: 10.1590/S0102$\underline{865020150120000010}$ 